\section{Concerning: Benson et al: Causes of Higher Blood Loss during General Anesthesia Compared to Spinal Anesthesia in Total Hip Replacement - a Retrospective Analysis of Data Collected Online. Infus Ther Transfus Med 2000;27:311-316.}

1) The conclusion of the authors that 'The analysis also shows that the clinical judgement of the anesthesiologist correlates well with laboratory parameters indicating blood loss' has to be challenged. A more appropriate conclusion from the results of the study would be that it is notoriously difficult to estimate blood loss in this setting and that blood loss is usually underestimated. The correlation between blood loss as estimated by the anesthesiologist and blood loss calculated may be significant $(p \leq 0.05)$, but it is weak $(r=0.23)$. For comparison: If a (new and less invasive) monitoring method is compared to a 'gold standard', a correlation of $r=0.8-0.9$ is usually required.

The formula used to calculate intraoperative blood loss, the amount of intraoperative blood loss calculated, and the time of transfusion are not stated in the paper. I assume that a linear formula was used, that the expansion of blood volume prior to surgery was not taken into consideration, and that the transfusion of red blood cells was carried out predominantly or exclusively in the postoperative period. In this case, the mean blood loss calculated by the authors can be estimated from the mean blood volume and mean pre- and postoperative hemoglobin level. It would amount to approximately 950 $\mathrm{ml}$. A more sophisticated approach would employ a linear equation for the expansion of blood volume due to volume resuscitation after induction of anesthesia and a logarithmic formula for normovolemic hemodilution during surgery $[1,2]$. If the initial volume expansion was somewhere close to $500 \mathrm{ml}$, a blood loss of approximately $900 \mathrm{ml}$ is computed. The amount of blood loss in such studies is usually not normally distributed [3-5]. The high values of the standard deviation (as compared to the mean) would suggest that for this investigation also. Thus, computations based on means will give a crude approximation only. They would, however, indicate that the estimation of blood loss by the anesthesiologist (mean $648 \mathrm{ml}$ ) falls short of the actual value by roughly $30 \%$. Regression analysis might have helped to identify the bias involved.

2) The relevance of the postoperative blood loss should have been discussed even if data are not available. The amount of red blood cells transfused $(569 \mathrm{ml}$ packed red blood cells, hematocrit 0.60) clearly exceeds the amount lost that is stated in the paper ( $648 \mathrm{ml}$ blood, mean hematocrit approximately $0.36)$. I would estimate from the data that approximately half of the total blood loss occurred postoperatively and that the average blood loss totalled some $1,800 \mathrm{ml}$. This is in agreement with other investigations [3-6].

3) The statement «In dieser Studie wurde eine längere Operationsdauer unter Allgemeinanästhesie als Grund für einen höheren Blutverlust festgestellt» or somewhat more cautious in the English summary 'In this study, duration of surgical procedure under general anesthesia as compared to spinal anesthesia was evaluated as being $a$ cause for increased blood loss' is not supported by the results of the study. A statistical correlation does not constitute a causation. Proving cause-and-effect relations is much more difficult than just showing a (high) correlation coefficient.

4) The statement 'This method (i.e. online documentation and retrospective analysis) makes it possible to carry out studies which would otherwise not be feasible either because of ethical reasons ...' seems to imply that major diagnostic or therapeutic conclusions may be derived from such studies. This is not the case. Retrospective studies provide only weak evidence for questions of scientific or clinical interest, unless appropriate sophisticated statistical techniques ('matched pairs', multivariate analysis) are used. Even then they cannot substitute prospective controlled randomized trials [7]. They are however valuable for the formulation of hypotheses for such trials as the authors state in the summary.

5) The authors used numerous statistical tests on the same set of data. It would have been helpful if they stated whether the $\alpha$ value was corrected for multiple testing, so the reader could assess the comparison-wise and investigation-wise error rate.

A. Lorentz, Mannheim

\section{References}

1 Bourke DL, Smith TC: Estimating allowable hemodilution. Anesthesiology 1974; 41:609-612.

2 Lorentz A, Gasteiger P, Osswald PM: Berechnung des zulässigen Blutverlustes vor Transfusion mit einem programmierbaren Taschenrechner. Anaesthesist 1987;36:306-312 (Erratum in Anaesthesist 1998;37:396).

3 Lorentz A, Schipplick M, Gmehlin U, Osswald PM, Winter M: Präoperative Eigenblutspende mit Flüssiglagerung bei künstlichem Gelenkersatz. Anaesthesist 1989;38:480-489.

4 Lorentz A, Homenou W, Schipplick M, Osswald PM, Winter M: Intra- und postoperative Autotransfusion in der Hüftgelenkschirurgie: Effektivität, Bakteriologie. Anästh Intensivther Notfallmed Schmerzther 1991;26:79-86.

\begin{tabular}{ll}
\hline KARGER & @ 2001 S. Karger GmbH, Freiburg \\
$\begin{array}{l}\text { Fax +49 7614520714 } \\
\begin{array}{l}\text { E-mail Information@Karger.de } \\
\text { www.karger.com }\end{array}\end{array}$ & Accessible online at: \\
www.karger.com/journals/iut
\end{tabular}


5 Lorentz A, Konermann W, Kellenbenz C, Heine T, Zimmermann G, Segiet W, Jani L: Mannheimer Konzept der präoperativen Eigenblutspende und perioperativen Autotransfusion in der Hüftgelenkendoprothetik. Z Orthop 2000;138: 311-317.

6 Toy PT, Kaplan EB, McVay PA, Lee SJ, Strauss RG, Stehling LC: Blood loss and replacement in hip arthroplasty: A multicenter study. The Preoperative Autologous Blood Donation Study Group. Transfusion 1922;32:63-67.

7 Spector R, Vesell ES: The pursuit of clinical truth: Role of epidemiology/observation studies. J Clin Pharmacol 2000;40:1205-1210.

\section{Authors' Reply}

First of all, we would like to express our thanks to A. Lorentz for his critical statement to our publication. This allows us to address misunderstandings in our examinations and to transmit a more exact definition of our study's goals to the interested reader.

1) Lorentz is correct in stating that the correlation between the estimated blood loss and the other indicators of blood loss is weak and cannot be compared to a 'gold standard'. We agree further with his remarks that it is difficult to estimate blood loss and that blood loss is underestimated. However, it was not the goal of our study to calculate blood loss as precisely as possible using online-documented data, as Lorentz expected. Rather, the aim of our study was, with the help of an anesthesia management system, to investigate factors that influence intraoperative blood loss during two types of anesthesia. The clinical estimation of intraoperative blood loss, performed by the anesthesiologist in charge, was our basis for providing an answer to this query. Laboratory parameters acted as additional variables of comparison and were not the basis for calculating blood loss. Although there is only a weak correlation between these values and the estimated blood loss, the responsible anesthesiologist's clinical judgment of the situation showed itself to be independent of these laboratory parameters. Blood loss can be assumed to be estimated at similar (low) levels during general and regional anesthesia. This underestimation most likely has no influence on our study's goal, namely to determine factors of influence on blood loss, independent of actual blood loss. These were not calculated based on available estimation equations, as Lorentz has suggested.

2) In addressing apparent misunderstandings, we would like to restate that our investigations were limited to the perioperative time range where the patient was in anesthesiological care. This time range generally ended with the recovery room, this also being the place where online documentation is completed. We were therefore unable to judge postoperative blood loss, although we realize that blood loss generally is comparably as high in the postoperative phase than in the intraoperative phase as Lorentz pointed out. Therefore, we cannot enter a serious discussion on postoperative blood loss even if Lorentz desired so.

3) We also agree with Lorentz when he states that a statistical correlation of duration of surgical procedure to higher esti- mated blood loss under general anesthesia does not constitute a causation. The formulization in our summary is rather unfortunate. However, we mention in the discussion that duration of surgery seems to be partly responsible for blood loss. This also has been confirmed by other studies. We discussed possible reasons for the influence of operation time on blood loss as well as reasons for a longer operation time. A prospective study would be beneficial in order to better judge the real influence of operation time as well as body temperature. We have planned to do that subsequent to this analysis.

4) We noted in the conclusion that with our method of retrospective analysis of online-documented data, we obtained results that are comparable to those of prospective studies making use of and reference to available literature in our discussion. It was our goal to show that the study gives useful insights into the routine procedure within a hospital, and the data can be used for internal quality assurance, helping to recognize deficits and aiding in the development and verification of quality improvement strategies. We have made a self-critical note of the fact that these online data collected in routine do not give the same objective comparison as does a prospective collection of complete and uniform study material data. We do not claim that this method can replace prospective studies, but it can be used to formulate a hypothesis or as a prompt to a planned prospective study. We allow ourselves to offer outlook on the fact that in some cases it could be an alternative to prospective studies when the quantity of the data is sufficient and the aim of the study and criteria for inclusion and exclusion as well are well-defined. This method makes it possible to carry out studies which would otherwise not be possible, either because of ethical reasons or because they include risks for the patients involved. Lorentz seems to have misunderstood us when he states that we will derive major diagnostic or therapeutic conclusions from such retrospective studies.

5) We would have liked to use statistical techniques used such as 'matched pairs' and multivariate regression analysis for our data analysis. However, our statisticians deemed data analysis in this form impossible due to the data structure. We had to limit ourselves to the analytical methods mentioned in the Methods section. We agree with Lorentz that it would have been helpful to correct the $\alpha$ value for multiple testing, which we haven't carried out.

We hope that with these responses, motivated by Lorentz, certain misunderstandings concerning our publication could be clarified. Using our examinations as an example, we wanted to demonstrate the possibilities of retrospective data analysis of online-documented anesthesia procedures. Even if it seems that we have not been able to be completely successful in this, we still have the opinion that this method offers alternatives to scientific queries, especially within the framework of quality assurance.

M. Benson, B. Hartmann, Gießen 